\title{
Recent Developments and Current Issues in the Treatment of Pancreatic Cancer
}

\author{
Helmut Oettle \\ Charité-Universitätsmedizin Berlin, Medizinische Klinik mit Schwerpunkt Hämatologie, Onkologie und Tumorimmunologie, Berlin, \\ Germany. \\ Email: helmut.oettle@charite.de
}

Received July $18^{\text {th }}, 2013$; revised August $15^{\text {th }}, 2013$; accepted August $24^{\text {th }}, 2013$

Copyright (C) 2013 Helmut Oettle. This is an open access article distributed under the Creative Commons Attribution License, which permits unrestricted use, distribution, and reproduction in any medium, provided the original work is properly cited.

\begin{abstract}
Presently, many questions exist about what the optimal regimen comprises for all stages and treatment settings for pancreatic cancer. Since the CONKO-001 trial, adjuvant therapy following resection has become standard of care; however, outcomes are poor, with most patients experiencing disease recurrence, and new therapies have yet to be validated. Furthermore, the value of adjuvant radiotherapy has still not been clearly defined. Targeted treatment in combination with chemotherapy has been mostly disappointing so far in the adjuvant setting but immunotherapy holds potential for improving survival outcomes. Neoadjuvant treatment does not appear to provide much benefit in resectable patients but in the small subgroup of patients with borderline resectable/unresectable locally advanced disease it may increase the possibility of an R0 resection and, consequently, a substantial increase in survival duration. Use of capecitabine-based radiotherapy in patients with unresectable locally advanced disease appears to be more efficacious and better tolerated than gemcitabine-based chemoradiotherapy, with respect to survival outcomes. However, as with adjuvant treatment, the benefit of adding radiotherapy has not yet been definitively demonstrated. In patients with metastatic pancreatic cancer, targeting the stroma with nab-paclitaxel has shown promising results in a phase III trial setting when administered in combination with gemcitabine and, furthermore, this regimen is suitable for a broad range of patients due to its generally good tolerability profile. Because of its high toxicity, FOLFIRINOX is more suitable for younger patients with an excellent performance status who can withstand aggressive treatment and in patients with a worse performance status, gemcitabine monotherapy is considered to be a more appropriate treatment. Alternatively, gemcitabine in combination with erlotinib, the only targeted compound that has resulted in significant albeit small improvements in survival in patients with advanced disease, could be selected. However, the benefit-risk profile of this regimen is only favorable in a strictly defined, small patient subgroup who develop a treatment-related rash. Finally, with the elucidation of prognostic and predictive markers, treatment is expected to become ever more individualized, leading to improved efficacy outcomes and less unnecessary toxicity.
\end{abstract}

Keywords: Pancreatic Cancer; Chemotherapy; Adjuvant Therapy; Neoadjuvant Therapy

\section{Introduction}

With a median survival duration of approximately 6 months following diagnosis and a 5-year survival rate of less than $5 \%$, pancreatic cancer is considered to have the poorest prognosis of any solid tumor [1]. Recent statistics show that pancreatic cancer is the fourth most common cause of death in the US and Europe and yet it accounts for only $3 \%$ of total cancer cases [2]. As well as displaying a highly drug- and radio-therapy resistant phenotype, pancreatic cancer has high potential for local invasion and metastasis to distant sites compared with other solid tumors [3]. Indeed, for the few patients who have seemingly resectable disease at diagnosis, distant micrometastases have usually already been established [2]. Moreover, due to its initially highly asymptomatic nature, more than $80 \%$ of patients only present once disease is advanced [4]. However, although progress in the treatment of pancreatic cancer in the past few decades has been incremental, it is nevertheless steadily increasing.

\section{Risk Factors}

Recently, a number of factors have been identified as 
having a possible causative role in the development of pancreatic cancer, including non-O blood group, diabetes mellitus, low vegetable intake and a high-fat diet; although most of these have yet to be validated as definitive risk factors [5]. However, a causative role for tobacco was determined several years ago, with smokers being found to have an elevated pancreatic cancer risk of 2.5 to 3.6 times that of a non-smoker [6]. Another well established risk factor is familial mutations, with approximately $5 \%$ - $10 \%$ of patients having a family history of pancreatic cancer [7]. An individual from a family with at least four affected members is approximately 57 times more likely to develop pancreatic cancer when compared with an individual from a family containing no affected members [4].

\section{Genetics}

Our understanding of pancreatic cancer genetics has increased in the last decade with a number of germline and aquired somatic mutations being identified and mapped. The vast majority of patients with pancreatic cancer from any cause carry at least one of four known mutations, with $90 \%$ of tumors carrying a mutation in the KRAS oncogene and $95 \%$ of tumors with inactivation of the CDKN2A gene which codes for p16, a regulator of the G1-S transition of the cell cycle [8]. In approximately $50 \%$ of tumors, the tumor suppressor gene DPC4 is lost and in $50 \%-75 \%$ of tumors, TP53, another tumor suppressor gene, is abnormal. Recently, whole genome sequencing of 24 pancreatic cancers revealed on average 63 mutations per cancer, implying that pancreatic cancer is a very complex and heterogeneous disease and consequently that it might respond best to a multi-pronged and/or individualized treatment approach [9].

\section{Tumor Microenvironment}

The importance of the microenvironment in tumor propagation has begun to be realized in recent years and is an area of great interest in the study of pancreatic cancer. A prominent feature of the tumour microenvironment in pancreatic cancer is the formation of a dense complex stroma, known as a desmoplastic reaction, around the tumour which can comprise up to $90 \%$ of the tumor volume [10]. This compact fibrous yet dynamic tissue functions as more than just a mechanical barrier, playing host to a complex interplay between normal host epithetlial cells, tumor cells, stromal fibroblasts, pancreatic stellate cells, endothelial cells and adipocytes, immune and inflammatory cells as well as growth factors which activate oncogenic signaling pathways [11]. There is now significant evidence that pancreatic stellate cells play a key role in stromal formation and turnover. Following activation by growth factors, pancreatic stellate cells se- crete collagen as well as other extracellular matrix (ECM) proteins $[12,13]$. Furthermore, pancreatic stellate cells contribute to the hypoxia/fibrosis cycle in the peritumoural stroma via abnormal ECM protein secretion and amplification of endostatin production by tumor cells [14]. They also regulate stromal turnover and reabsorption, mostly through generation of matrix metalloproteinases. Proteins produced by stromal cells and associated with poor prognosis to treatment include stromal cell-derived factor, chemokines, cyclooxygenase-2, PDGF receptor, hedgehog pathway elements, vascular endothelial growth factor, integrins, and SPARC (Secreted Protein Acidic And Rich in Cysteine) [4]. As a consequence of our increased understanding of the tumour microenvironment, new therapeutic opportunities have arisen.

For example, the nanoparticle albumin-bound (nab)paclitaxel is able to bind with SPARC, which is found in abundant levels in the stroma, resulting in increased delivery of paclitaxel to tumor cells [15]. Another emerging area of research is of the small subset of cells within a tumor termed stem cells. Pancreatic stem cells make up approximately $0.5 \%-1 \%$ of all tumour cells and express the protein markers CD44, CD24 and epithelial-specific antigen [16]. Responsible for tumor initiation and propagation, they are also hypothesized to contribute to metastasis although, thus far, solid evidence is lacking in this area. However, stronger evidence has come to light for a role of stem cells in the typically high resistance displayed by pancreatic cancer towards chemotherapy and radiotherapy. In an in-vitro study [17], exposure to gemcitabine resulted in an enriched population of CD133+ cells in the $13.6 \mathrm{p}$ pancreatic cancer cell line and in another in-vitro study [16], exposure to gemcitabine and ionising radiation resulted in an enriched population of CD44, CD24, and ESA in xenografts of human pancreatic cancer. However, although our understanding of the molecular processes that underpin the development and propagation of pancreatic cancer has deepened in recent years, translation of this knowledge into effective therapies has yet to be realized.

\section{Adjuvant Therapy}

\subsection{Chemotherapy/Chemoradiotherapy}

Currently, surgical resection is the only curative treatment for stage I/II pancreatic cancer (Figure 1). Nevertheless, following surgery, rates of locoregional and distant recurrence are high, occurring in $50 \%-80 \%$ and more than $70 \%$ of patients, respectively. As a result, 5year survival rates following surgery with a curative intent are low, ranging from $10 \%-15 \%[18]$ and, consequently, adjuvant therapy is considered an important facet of treatment for early-stage pancreatic cancer. Although accepted now as standard of care, for a long time 


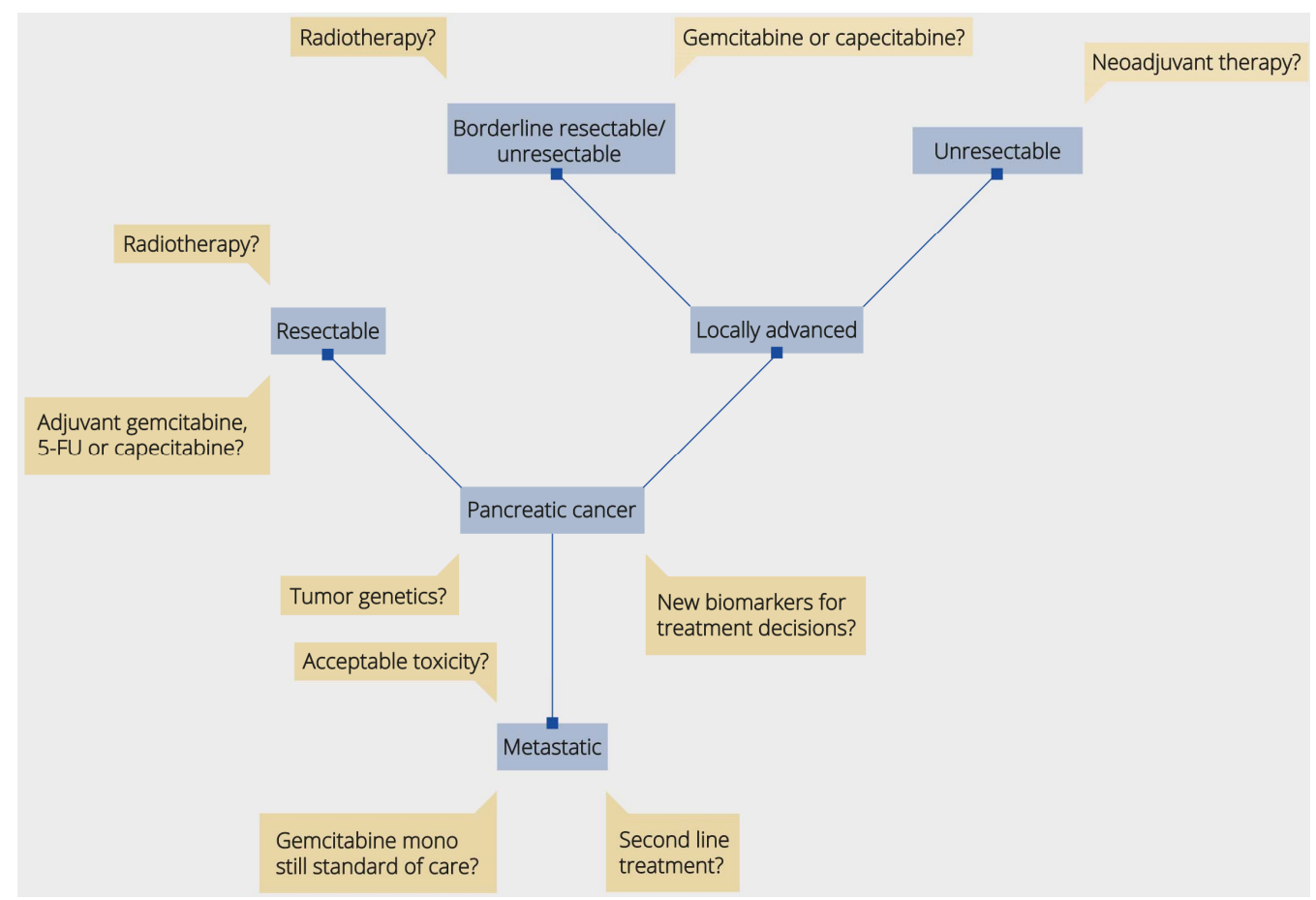

Figure 1. Current issues in pancreatic cancer treatment.

the benefits of administering adjuvant chemotherapy with or without radiotherapy following resection were unclear. In trials conducted in the late 1960s and early 1970s, patients with advanced unresectable pancreatic cancer received external beam radiotherapy with or without systemic chemotherapy $[19,20]$. As a result of the antitumour activity observed in these studies, the first randomized trial of adjuvant therapy was initiated by the Gastrointestinal Tumour Study Group (GITSG) in which patients undergoing resection with curative intent received 5-FU + folinic acid administered with radiotherapy or underwent observation alone [21]. Significantly ( $p$ $=0.03$ ) longer median survival durations were observed in patients who had received adjuvant therapy, compared with those who had not (20 vs. 11 months) [21]. Unfortunately, this trial was found to have multiple shortcomings and reliable conclusions could not be drawn from it [2]. In response to these criticisms, the GITSG conducted a single-arm study $(\mathrm{n}=30)$ comprising the same adjuvant treatment protocol which demonstrated similar results [22]. Adjuvant therapy therefore appeared to be beneficial although it still remained unclear as to whether chemotherapy alone or chemoradiotherapy was responsible for these improvements in survival. Following the GITSG trial, two pivotal trials, (EORTC-40891 and ESPAC-1) were conducted which showed contradictory outcomes. In the EORTC-40891 trial, 5-FU + folinic acid-based chemoradiotherapy did not significantly improve overall survival at 5 years when compared with observation alone in the overall patient population which included patients with periampullary tumors (median of 24.5 vs. 19.0 months) yet a trend $(\mathrm{p}=0.099)$ towards a survival benefit was observed in patients with pancreatic head cancer (median survival duration of 17.1 vs. 12.6 months) [23]. Moreover, a long-term survival analysis also showed no significant benefit of adjuvant treatment in the overall patient population, with median survival durations of 1.8 and 1.6 years in the chemoradiotherapy and observation alone arms, respectively [24]. However, due to inadequate statistical power, one of the numerous shortcomings of the EORTC-40891 trial, a possible survival advantage of adjuvant chemoradiation could not be definitively ruled out. In the ESPAC-1 trial, which utilized a $2 \times 2$ factorial design, 5-FU + folinic acid-based chemoradiotherapy actually had a deleterious effect on survival, resulting in a significantly $(\mathrm{p}=0.05)$ lower survival rate at 5 years, compared with no chemoradiotherapy ( $10 \%$ vs. $20 \%$ of patients). However, adjuvant $5 \mathrm{FU}+$ folinic acid was found to be significantly $(\mathrm{p}=0.009)$ more efficacious than no adjuvant 5-FU + folinic acid, with respective survival rates at 5 years of $21 \%$ and $8 \%$ [25].

Due to these conflicting outcomes, the benefit of adjuvant therapy remained debatable, especially with respect to addition of radiotherapy to chemotherapy. However, subsequent publication of several larger and more well-designed landmark clinical trials has shed more light on this issue.

Due to its proven efficacy in the palliative setting, gemcitabine was investigated as a possible adjuvant ther- 
apy in several phase III studies. In the CONKO-001 study, we randomly assigned patients to receive adjuvant gemcitabine or to undergo observation alone [26]. A significant $(p<0.001)$ and clinically relevant benefit was seen in the patients receiving adjuvant therapy, with a median disease-free survival interval of 13.4 months being achieved, compared with 6.9 months in the treatment group undergoing observation alone. Upon subgroup analysis, significant benefit was seen across all subgroups, including in patients with an R1 resection (15.4 vs. 5.5 months; $\mathrm{p}<0.001)$, who were node-negative (22.4 vs. 10.4 months; $p=0.006$ ) or with tumor stage T1-2 (27.5 vs. 10.0 months; $\mathrm{p}<0.05$ ). Interestingly, gemcitabine recipients with an R1 resection survived for longer disease-free than gemcitabine recipients who had achieved an R0 resection (median disease-free survival interval of 15.4 vs. 13.1 months). As well as prolonging disease-free survival, adjuvant gemcitabine also significantly ( $<<0.01$ ), albeit to a lesser degree, improved the hard endpoint of overall survival, with final results showing gemcitabine recipients surviving for a median of 22.8 months, compared with 20.2 months in the observation alone arm [26]. Again, significant benefit was observed in gemcitabine recipients who had undergone an R1 resection, with overall survival in this group being almost as long as gemcitabine recipients who had undergone an R0 resection (median of 22.1 vs. 22.8 months). This reduction in the magnitude of benefit from gemcitabine treatment in the overall patient population may have been due to the majority of patients under observation alone subsequently crossing over to the gemcitabine arm upon progression. With respect to the resection margin, significant $(p<0.05)$ benefit in overall survival remained in patients who had undergone an $\mathrm{R} 0$ resection (median of 22.8 vs. 20.3 months) whereas only a trend towards improved overall survival was observed in patients who had undergone an R1 resection (median of 22.1 vs. 14.1 months) [26].

The large ESPAC-3 version 2 trial $(n=1088)$ [27] was subsequently designed to compare the efficacy of adjuvant 5-FU + folinic acid, already established as standard of care for advanced pancreatic cancer, with gemcitabine which had earlier been validated as adjuvant therapy in the CONKO-001 trial. There had initially been an arm undergoing observation alone but this was discontinued prematurely due to the final results of ESPAC-1 demonstrating a benefit for adjuvant therapy. The main aim was to determine whether gemcitabine would result in an improvement in overall survival. However, no significant difference between the gemcitabine and 5-FU + folinic arms was observed in median overall survival duration, median progression-free survival duration or quality of life. Gemcitabine appeared to be better tolerated than 5 -FU + folinic acid which had resulted in significantly ( $p$
$<0.001$ ) increased rates of grade 3 or 4 stomatitis, grade 3 and 4 diarrhea and serious adverse events [27].

These results were in contrast to an earlier smaller study [28] in which gemcitabine resulted in significantly $(\mathrm{p}<0.01)$ greater antitumour activity as well as a significantly $(\mathrm{p}<0.01)$ higher overall survival rate at 2 years when compared with fluorouracil. However, the dose of 5-FU used in this earlier study was lower than the fluorouracil dose used in ESPAC-3 version 2.

The benefit of using gemcitabine as part of adjuvant chemoradiotherapy was again left unanswered in a trial conducted by the Radiation Therapy Oncology Group (RTOG-9704) [29]. In this trial, patients were randomly assigned to receive either adjuvant gemcitabine or 5-FU (control), both administered before and after 5-FU-based chemoradiotherapy. No significant difference in the primary endpoint of overall survival was reported between the treatment arms. However, in patients with cancer of the pancreas head, gemcitabine resulted in a trend $(\mathrm{p}=$ 0.09 ) towards a longer overall survival duration (median of 20.5 vs. 16.9 months), upon multivariate analysis.

Use of S-1 (gimeracil/oteracil/tegafur) as adjuvant therapy was explored by the Japan Adjuvant Study Group of Pancreatic Cancer in the JASPAC 01 trial [30]. In this noninferiority study, Japanese patients were randomised to S-1 or gemcitabine with the aim of determining whether S-1 would be any less efficacious than gemcitabine as adjuvant therapy. S-1 was found to be not only significantly $(\mathrm{p}<0.001)$ noninferior to gemcitabine, but was also found to be significantly $(p<0.001)$ superior, with respect to overall survival at 2 years $(70 \%$ vs. $53 \%$ in the full analysis set; HR $0.56 ; 95 \%$ CI 0.36 0.87 ).

Due to demonstrating efficacy in patients with advanced pancreatic cancer, capecitabine is being explored as a possible option in adjuvant therapy. In the currently recruiting ESPAC-4 trial (EudraCT2007-004299-38), patients with resectable pancreatic cancer or periampullary cancer are being randomised to gemcitabine + capecitabine or gemcitabine monotherapy. Completion is scheduled for November 2014.

Addition of radiotherapy to adjuvant chemotherapy remains controversial, with some studies reporting a clinical benefit but others, such as the ESPAC-1 trial, showing a deleterious effect [25]. Results from RTOG9704 and CONKO-001 seem to suggest that adjuvant chemoradiotherapy is no more efficacious than adjuvant chemotherapy $[26,29]$. However, this should be interpreted cautiously as the patient populations in these trials differed, with CONKO-001 having fewer patients with margin- or node-positive disease as well as an inclusion criterion of patients with CA19-9 concentrations of 90 $\mathrm{U} / \mathrm{mL}$ or less. In an exploratory subgroup analysis from a meta-analysis conducted by Stocken et al., chemoradio- 
therapy was found to be significantly more effective than chemotherapy in patients with positive (R1) resection margins [31]. For most oncology practices in Europe adjuvant chemotherapy alone is considered standard of care and adjuvant chemoradiotherapy is generally not administered whereas in the US adjuvant chemoradiotherapy and subsequent chemotherapy is the standard of care [32].

\subsection{Targeted Therapies}

After showing a small but significant benefit in overall survival in the phase III PA.3 trial [33] in advanced pancreatic cancer patients, erlotinib is being assessed as part of gemcitabine-based adjuvant therapy in the CONKO005 trial (EudraCT2007-003813-15) in patients who have undergone an $\mathrm{R} 0$ resection. In another study currently being conducted by the Charité Oncology Group (CONKO-006; EudraCT2007-000718-35), the multikinase inhibitor sorafenib is being explored as part of combination adjuvant therapy with gemcitabine, compared with gemcitabine alone, in patients with an R1 resection of pancreatic cancer. The benefit of adjuvant gemcitabine in patients with an $\mathrm{R} 1$ resection had already been demonstrated in subgroup analysis of CONKO-001, in which gemcitabine recipients with an $\mathrm{R} 1$ resection survived for almost 10 months longer than patients with an R1 resection who were under observation alone. Therefore, the aim of this study is to determine whether addition of sorafenib could increase disease-free survival (primary endpoint) and subsequently overall survival (secondary endpoint) in this patient subgroup. Duration of therapy will also be assessed, with patients being treated for one year in the CONKO-006 study, compared with only six months in the CONKO-001 and CONKO005 studies.

\subsection{Immunotherapy}

Immunotherapy is a therapeutic field that may hold great potential in the adjuvant setting, with the main aims being to inhibit regulatory T-cells which suppress the immune response to pancreatic cancer or to prime the immune system to recognize cancer cells via immunostimulatory pancreatic cancer antigens or use of genetically-modified irradiated pancreatic cancer cells [34]. Algenpantucel-L, which is the most clinically advanced pancreatic cancer vaccine currently in development, comprises an irradiated live combination of two human pancreatic cancer cell lines which present a non-human surface epitope (alpha-galactosyl), thus stimulating an immune response. A multi-institutional single-arm phase II trial assessed the efficacy of algenpantucel-L, when added to standard of care (adjuvant chemotherapy or chemoradiotherapy with gemcitabine or 5-FU) [35]. In this trial, the one-year survival rate was higher than that observed in the RTOG-9704 trial (86\% vs. 69\% of patients), even though lymph node involvement was higher at baseline ( $81 \%$ vs. $68 \%$ positive nodes). Furthermore, algenpantucel-L was well tolerated [35]. Based on these encouraging data, the randomised IMPRESS trial by Fisher et al. (NCT01072981) was initiated in April 2010 and is scheduled to be finished by January 2014. In this study, 922 patients will receive standard of care with or without algenpantucel-L [34]. In a phase II single-centre trial, a granulocyte-macrophage colony stimulating factor-transduced allogeneic whole cell vaccine was administered following adjuvant fluorouracil-based chemoradiotherapy. Disease-free survival and overall survival rates at one year were $67 \%$ and $85 \%$, respectively. Tolerability was good, with the most frequently reported adverse events being transient injection-site reactions [36]. Other vaccines containing ras-peptide, telomerase peptide, mucin + carcinoembryonic antigen and survivin are also in early-phase development as are vaccines comprising antigen-pulsed dendritic cells presenting either mucin 1 or carcinoembryonic antigen [34].

\section{Neoadjuvant Therapy}

As a result of waiting list times for surgery and the subsequent time needed for postoperative recovery, the time between diagnosis and receipt of chemotherapy can be 2 months or more. Due to micro-metastases in lymph nodes, lung, peritoneum and liver being present at diagnosis in the majority of patients, a rationale exists for the use of neoadjuvant therapy. Furthermore, as patients are at an earlier disease stage and therefore have an improved performance status, it is more likely that they will be eligible for treatment, compared with patients waiting to undergo adjuvant therapy. Neoadjuvant treatment may also reduce the risk of peritoneal tumor cell implantation during surgery due to decreasing intraoperative tumor spillage and may also result in more definitive surgical resections [37].

Unfortunately, clinical outcomes for neoadjuvant therapy have mostly been disappointing although positive results were shown in a small phase II study [38] in which 28 patients with resectable adenocarcinoma of the pancreatic head received 4 courses of neoadjuvant gemcitabine + cisplatin. Resection rate was $93 \%$, above that of the predetermined primary endpoint of at least $70 \%$ and improvements in nutritional status and quality of life were observed. Another phase II study [39] compared gemcitabine monotherapy with gemcitabine + cisplatin. Resection rates were $38 \%$ and $70 \%$, respectively, but survival outcomes were poor, with patients only surviving a median of 9.9 and 15.6 months, respectively. Administration of subsequent chemoradiotherapy following 
initial neoadjuvant chemotherapy has also been assessed in the phase II setting. One phase II trial [40] assessed gemcitabine then gemcitabine-based radiotherapy in 20 patients and another phase II trial [41] assessed cisplatin + gemcitabine then gemcitabine + radiotherapy in 90 patients. Disappointingly, survival outcomes were generally no better than those observed with surgery alone. Furthermore, in a meta-analysis [42] comprising 4394 patients, resection rates in patients with initially resectable tumours were found to be the same, regardless of whether or not patients received neoadjuvant treatment. Therefore, although there is a hypothetical rationale for use of neoadjuvant therapy in patients with resectable pancreatic cancer, stronger clinical data are needed to support its use in standard practice.

Currently, a neoadjuvant regimen comprising nab-paclitaxel and gemcitabine is being explored in a phase II pilot trial [43]. Preliminary results were presented at ASCO 2013 in which this regimen was determined as being feasible and warranting study in a larger trial.

\section{Borderline Resectable/Unresectable Pancreatic Cancer-A New Indication?}

Patients with borderline resectable/unresectable locally advanced pancreatic cancer constitute a unique patient population. In patients who are borderline resectable, neoadjuvant treatment offers an increased chance of an R0 resection and in those patients who are borderline unresectable, neoadjuvant treatment offers an increased chance of resection, including the possibility of an R0 resection and, consequently, for both patient populations, a much extended survival duration. In the previously mentioned meta-analysis by Gillen et al. [42], although benefit from neoadjuvant therapy was not demonstrated in patients with resectable tumours, approximately one third of patients who had initially unresectable tumours at baseline were able to undergo resection following neoadjuvant therapy, especially if combination chemotherapy was administered. Survival in this group was similar to that observed in patients with initially resectable tumours. A large retrospective series at a single institution subsequently confirmed these data [44]. Patients with locally-advanced unresectable pancreatic cancer received neoadjuvant gemcitabine-based chemoradiotherapy. Of the 215 patients studied, $26 \%$ were able to undergo secondary resection, with the median overall survival duration being substantially longer in the $36 \%$ of resected patients who underwent an R0 resection, compared with those patients who did not undergo any resection at all (22.1 vs. 11.9 months). Moreover, in an analysis of patients undergoing curative pancreatoduodenectomy or total pancreatectomy [45], patients requiring a portal/superior mesenteric vein resection had a signifi- cantly $(\mathrm{p}<0.05)$ shorter mean survival time, compared with patients who did not require this resection. However, adjuvant gemcitabine improved the prognosis of these patients such that the mean survival time was similar between these 2 groups of patients.

\section{Unresectable Locally Advanced Pancreatic Cancer}

\subsection{Chemoradiotherapy}

For patients with locally advanced unresectable disease, treatment options, including whether or not to administer radiotherapy, are not yet clearly defined. In a phase III study [46], chemoradiotherapy comprising fluorouracil + cisplatin and a total radiotherapy dose of 60Gy resulted in a significantly $(p=0.03)$ shorter median overall survival duration, compared with gemcitabine alone (median of 8.6 vs. 13.0 months), as well as increased moderate-to-severe toxicity. Yet in a subsequent phase III trial conducted by the Eastern Cooperative Oncology Group [47], chemoradiotherapy comprising gemcitabine (600 $\mathrm{mg} / \mathrm{m}^{2} /$ week) and radiotherapy administered to a total dose of 50.4 Gy was found to significantly $(p=0.017)$ improve overall survival over gemcitabine alone (median of 11.1 vs. 9.2 months). Unfortunately, due to limited statistical power resulting from poor patient accrual, this study was terminated prematurely and was not able to provide definitive evidence that could impact standard of care.

The SCALOP trial [48] was the first randomized multicenter trial to compare capecitabine-based chemoradiotherapy with gemcitabine-based chemoradiotherapy, both administered following induction treatment with gemcitabine + capecitabine, in patients with locally advanced disease. Radiotherapy was 3D conformal or intensitymodulated and was administered as 5.5 fractions per week to a total dose of $50.4 \mathrm{~Gy}$, although only $68 \%-69 \%$ of patients received the full protocol dose. Progressionfree survival rates at 9 months (primary endpoint) were $63 \%$ and $51 \%$, respectively, which met prespecified criteria according to a Fleming's design. However, capecitabine resulted in a non-significantly $(p=0.111)$ longer median progression-free survival interval, compared with gemcitabine (12.0 vs. 10.4 months; HR $0.60 ; 95 \% \mathrm{CI}$ 0.32 - 1.12), including a longer median local progression-free survival interval (14.6 vs. 12.0 months) as well as a longer distant progression-free survival interval (14.3 vs. 11.9 months). More importantly, capecitabine was found to significantly $(p=0.012)$ extend overall survival, compared with gemcitabine (median of 15.2 vs. 13.4 months; HR $0.39 ; 95 \%$ CI $0.18-0.81$ ). With respect to secondary endpoints, 2 patients $(6 \%)$ in the capecitabine arm alone experienced a complete response but, in general, response rates were similar between treatment 
arms. Of the $58 \%$ of patients with progression in each treatment arm, 33\% and 32\%, respectively, experienced local relapse, $52 \%$ and $46 \%$, respectively, experienced metastatic relapse and $14 \%$ and $23 \%$, respectively, experienced both types of relapse. Following radiotherapy, similar numbers of patients were eligible for resection ( $6 \%$ vs. $8 \%$ of patients, respectively). Furthermore, capecitabine resulted in fewer grade 3 and 4 adverse events, compared with gemcitabine (11\% vs. $37 \%$ of patients), including significantly $(\mathrm{p}=0.007)$ fewer grade 3 and 4 hematological adverse events ( $0 \%$ vs. $18 \%$ of patients) and a trend $(\mathrm{p}=0.095)$ towards fewer grade 3 and 4 non-hematological adverse events (11\% vs. $26 \%$ of patients) [48].

The $2 \times 2$ factorial LAP07 study [49] aimed to compare switching to capecitabine-based chemoradiotherapy with continuing either erlotinib + gemcitabine or gemcitabine monotherapy. Patients who had received erlotinib during the initial induction treatment could continue to receive erlotinib as maintenance therapy, regardless of whether or not they were randomized to continue treatment. Disappointingly, no significant difference in the primary endpoint of overall survival was observed between the chemoradiotherapy and the chemotherapy arms (15.3 vs. 16.5 months; HR 1.03; 95\% CI 0.79 - 1.03) and nor was there any significant difference in progression-free survival (HR 0.9; 95\% CI 0.7 - 1.1).

Comparison of chemoradiotherapy versus chemotherapy alone following induction with either gemcitabine, gemcitabine + nab-paclitaxel or 5 -FU + folinic acid + irinotecan + oxaliplatin (FOLFIRINOX) is being investigated in the CONKO-007 trial by Fietkau and Oettle (NCT01827553). Following completion of radiotherapy, patients will continue to receive the same chemotherapy regimen as they received for induction therapy until disease progression. Overall survival is the primary endpoint and resectability status is being assessed as a secondary endpoint.

\subsection{Investigational Agents in Locally Advanced Pancreatic Cancer}

Gene therapy was recently assessed in a phase III trial in which golnerminogene pradenovec, a genetically-modified adenovirus 5 vector encoding tumour necrosis factor-alpha, was administered with 5-FU + radiotherapy and compared with 5-FU + radiotherapy alone. Nonsignificant improvement in the primary endpoint of overall survival was observed in the investigational treatment $\operatorname{arm}[50]$.

\section{Metastatic Pancreatic Cancer}

\subsection{First-line Therapy}

Gemcitabine has comprised the first-line standard of care for metastatic pancreatic cancer since 1997 when a pivotal study by Burris et al. demonstrated superiority of gemcitabine over 5-FU [28]. Subsequently many different combinations using gemcitabine as a backbone have been evaluated including 5-FU [51], capecitabine [52], oxaliplatin [53], cisplatin [54], irinotecan [55] and pemetrexed [56]. Overall, these combination regimes failed to demonstrate significant prolonged survival as compared to gemcitabine alone although a significant but modest benefit in survival of gemcitabine-based combination regimens in patients with good performance status was demonstrated in 2 meta-analyses [57,58].

\subsection{Targeted Therapies}

Although our understanding of the molecular and genetic changes associated with the development and propagation of pancreatic cancer has increased over recent years, there is still a lack of suitably targeted drugs for this disease. Despite demonstrating efficacy in other solid tumours, targeted therapies have so far resulted in disappointing outcomes in advanced pancreatic cancer. The only targeted compound which has demonstrated prolonged survival in a phase III clinical trial setting was erlotinib when administered in combination with gemcitabine [33]. In the phase III PA.3 trial, erlotinib in combination with gemcitabine resulted in a small but significantly $(p<0.05)$ greater extension in overall survival, compared with gemcitabine alone (HR 0.82; median of 6.24 vs. 5.91 months). However, as this increase was not considered clinically relevant by most oncologists erlotinib has not been broadly adopted as part of standard of care. Other targeted therapies in combination with gemcitabine that have been investigated, including monoclonal antibodies such as bevacizumab and cetuximab or antiangiogenic multikinase inhibitors such as axitinib and sorafenib, have failed to show survival benefit in advanced pancreatic cancer [59-63]. Development of the once-promising hedgehog signaling pathway inhibitor vismodegib (GDC 0449) for the treatment of advanced pancreatic cancer was discontinued recently due to unfavorable results.

Many other targeted therapies are currently being investigated in early-phase clinical trials, including the hypoxia-targeted drug TH-302 [NCT01746979], the antiIGF-R1 antibody MK 0646 [NCT00769483], and the PARP inhibitor ABT-888 [NCT01489865], although phase III confirmatory studies will be needed to definitively prove the clinical benefit of these and other agents.

\subsection{Polychemotherapy with FOLFIRINOX}

Substantial progress in the treatment of advanced pancreatic cancer has been made by the introduction of the FOLFIRINOX regimen (oxaliplatin, irinotecan fluorouracil, and leucovorin) [64]. In this phase III clinical trial, which 
compared FOLFIRINOX with the current treatment standard gemcitabine, patients receiving FOLFIRINOX benefited significantly in median progression-free survival (6.4 months vs. 3.3 months, $p<0.001)$ and also in the primary endpoint of overall survival (11.1 months vs. 6.8 months, $\mathrm{p}<0.001)$. However, it should be noted that FOLFIRINOX resulted in higher toxicity as compared to gemcitabine. Specifically, patients presented with more grade 3 or 4 neutropenia $(45.7 \%$ vs. $21.0 \%$, p $<0.001)$ and febrile neutropenia ( $5.4 \%$ vs. $1.2 \%)$, which required use of granulocyte-colony stimulating factor. The tolerability profile of FOLFIRINOX was also less favorable in terms of grade 3 or 4 thrombocytopenia $(9.1 \%$ vs. $3.6 \%, \mathrm{p}=0.04)$, grade 3 or 4 diarrhea $(12.7 \%$ vs. $1.8 \%, \mathrm{p}$ $<0.001)$ and grade 3 or 4 sensory neuropathy $(9.0 \%$ vs. $0 \%, \mathrm{p}<0.001)$. Interestingly, despite the increased toxicity of FOLFIRINOX regimen, time to deterioration in quality of life was similar to that observed with gemcitabine. However, since inclusion critera were strict (patients had to be younger than 76 years with a high performance status [ECOG 0 or 1] with no cardiac ischemia and good hepatobiliary function) this does not represent a real life setting and subsequently the clinical impact of this study is thought to be modest. Moreover, FOLFIRINOX has not been readily adopted by oncologists practice due to general safety concerns about the risk of cholangitis in stented patients [65]. Currently, a modified FOLFIRINOX regimen with expected reduced toxicity due to omission of the 5-FU bolus (FOLFOXIRI) is being investigated [66].

\subsection{Targeting the Stroma with Nab-Paclitaxel}

A unique feature of pancreatic cancer is an abundant stroma which impairs drug delivery by reducing drug diffusion into the primary tumour. This is thought to be a major factor in the notable resistance of pancreatic cancer to chemotherapy [67] and, consequently, many researchers have tried to improve drug delivery by targeting the stroma of pancreatic cancer cells. A recent breakthrough in this area is nanoparticle albumin-bound (nab)paclitaxel that was initially developed to reduce treatment toxicity by allowing omission of the solvent needed to dissolve oily drug formulations of paclitaxel. Pancreatic peritumoral fibroblasts cancers overexpress SPARC [68], which serves, via mediation of the albumin-binding protein gp60, as a strong binding protein for nab-paclitaxel [69] (Figure 2). Von Hoff et al. demonstrated convincing evidence of this principle in a phase I/II trial, with a response rate to nab-paclitaxel of $48 \%$, a median overall survival (OS) of 12.2 months and 1-year survival rate of $48 \%$ [70]. They also reported interesting preclinical data; most importantly stroma depletion resulted in a 2.8-fold higher intratumoral gemcitabine concentration in mice receiving nab-paclitaxel plus gemcitabine when compared to gemcitabine alone. At this years ASCO gastrointestinal cancers symposium, Von Hoff et al. presented the results of a phase III trial in 861 patients (MPACT), which confirmed the phase II data [71]. Nabpaclitaxel plus gemcitabine was superior to gemcitabine for all efficacy endpoints with a median overall survival of 8.5 vs. 6.7 months (HR $0.72 ; p=0.000015$ ), a median progression free survival of 5.5 vs. 3.7 months (HR 0.69; $\mathrm{p}=0.000024)$, an investigator-assessed overall response rate of $29 \%$ vs. $8 \%\left(p=3.3 \times 10^{-16}\right)$, and an improved one-year overall survival of $35 \%$ vs. $22 \%(p=0.0002)$. These improvements in efficacy for nab-paclitaxel plus gemcitabine were accompanied by good tolerability data. With comparable dose intensities being administered in both treatment arms, grade 3 or higher adverse events with nab-paclitaxel comprised neutropenia (38\% vs. $27 \%$ ), febrile neutropenia (3\% vs. $1 \%$ ), thrombocytopenia (13\% vs. $9 \%)$, anemia (13\% vs. $12 \%)$ fatigue $(17 \%$ vs. $7 \%)$, and diarrhea (6\% vs. $1 \%)$. Grade 3 or higher peripheral neuropathy occurred more often with nab-paclitaxel plus gemcitabine (17\% vs. $1 \%$ ) but improved to no more than grade 1 over a median period of 29 days [71].

\subsection{Is Nab-Paclitaxel a New Treatment Standard?}

Despite the inherent limitations of inter-study comparison, the tabular overview (Tables 1-3) shows that in comparison to FOLFIRINOX, the nab-paclitaxel regime provides similar efficacy to FOLFIRINOX but with better tolerability. In contrast to FOLFIRINOX, nab-paclitaxel + gemcitabine was better tolerated even though the patient population included less fit patients. Notably, nab-paclitaxel + gemcitabine resulted in a lower rate of grade 3 and grade 4 neutropenia, compared with FOLFIRINOX (45.7\% vs. 38\%). Grade 3 and 4 neuropathy was found to be higher with nab-paclitaxel (17\% vs. $9 \%$ ), but improved quickly within 29 days to no more than grade 1 [64,71]. Looking at different subgroups patients of the MPACT trial upon multivariate analyses it was revealed that patients with a Karnofsky performance status of 70 80 (HR 0.61), one metastatic site (HR 0.41), more than 3 metastatic sites (HR 0.5) and increased ( $\geq 59 \times$ ULN) CA-19-9 (HR 0.61) particularly benefited from nab-paclitaxel + gemcitabine [72]. In comparison with gemcitabine alone [28] and gemcitabine + erlotinib [33], nabpaclitaxel + gemcitabine resulted in a substantial benefit in overall survival ( 8.5 month vs. 6.24 month vs. 5.65 month), mitigating the increased toxicity of nab-paclitaxel. In conclusion, nab-paclitaxel + gemcitabine has a favorable toxicity profile and is a potentially new standard therapy for the treatment of metastatic pancreatic cancer in a broad range of patients. FOLFIRINOX should be restricted to younger patients who have an excellent performance status and are willing to undergo 


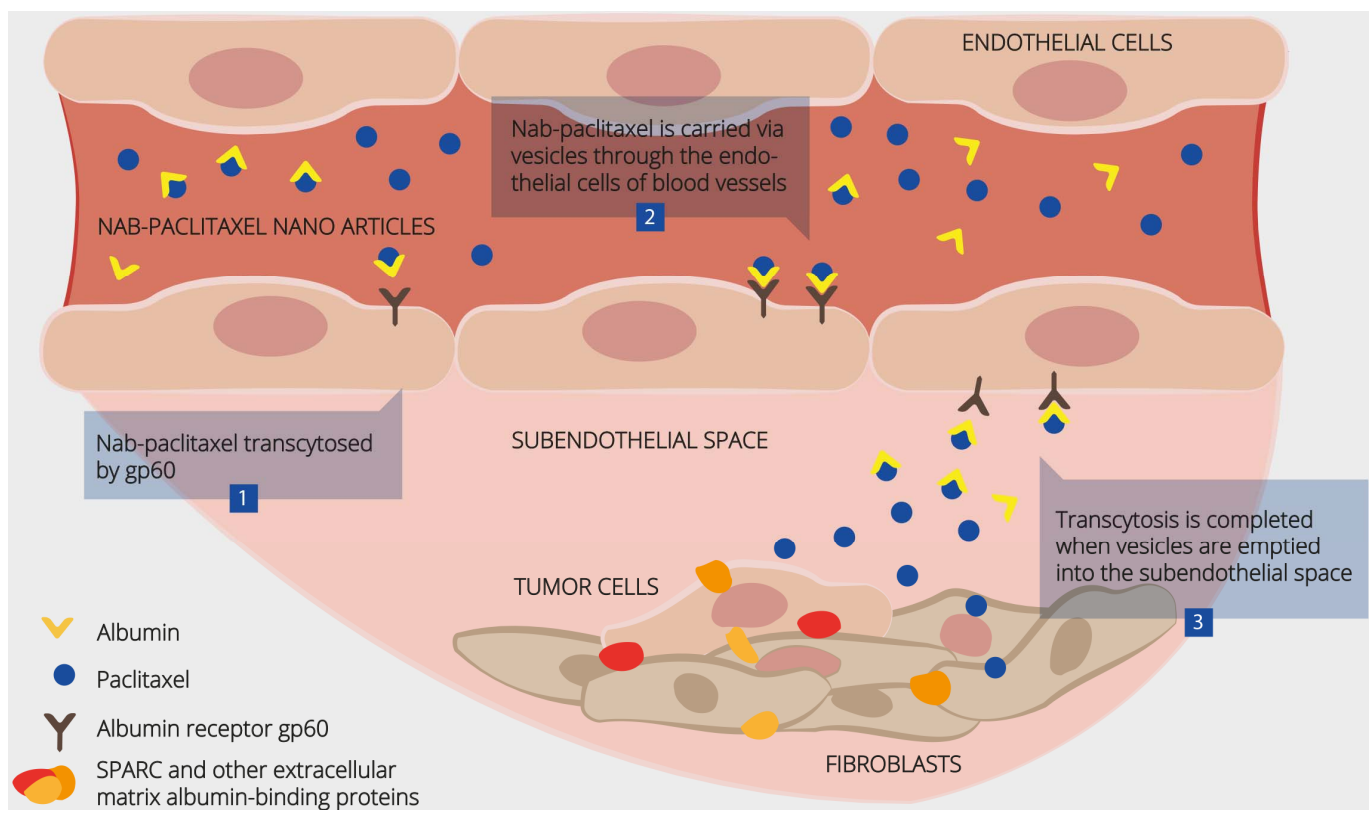

Figure 2. Mechanism of nab-paclitaxel drug-delivery.

Table 1. Patient demographics in selected pivotal phase III trials in patients with advanced disease.

\begin{tabular}{ccccc}
\hline Study & GEM [28] & GEM/ERL [33] & FOLFIRINOX [64] & NABPAC [71] \\
\hline Age (range) & $62(37-79)$ & $63.7(37-84)$ & $61(25-76)$ & $62(27-88)$ \\
& & $29.8 \%$ ECOG 0 & $37.4 \%$ ECOG 0 & $58 \%$ KPS $90-100$ \\
Performance status & $30 \%$ KPS $80-90$ & $50.9 \%$ ECOG 1 & $61.9 \%$ ECOG 1 & $42 \%$ KPS $70-80$ \\
& $70 \%$ KPS $50-70$ & $18.9 \%$ ECOG 2 & $0.6 \%$ ECOG 2 & $85 \%$ \\
Liver metastases & NR & NR & $87.6 \%$ & $39 \%$ \\
Head of pancreas & NR & NR & $39.2 \%$ & $46 \%$ \\
CA19.9 $\geq 59$ ULN & NR & NR & $41.5 \%$ & $46 \%$ \\
\hline
\end{tabular}

GEM, gemcitabine; ERL, erlotinib; NABPAC, nab-paclitaxel; KPS, Karnofsky performance status; ECOG, Eastern Cooperative Oncology Group performance status; ULN, upper limit of normal; NR, not reported.

more aggressive treatment. In patients with a Karnofsky performance status of $<70 \%$, gemcitabine monotherapy can be administered, if applicable with erlotinib being added but then subsequently discontinued if no rash has developed within the first 10 - 15 days.

\subsection{Second-Line Therapy}

There is no generally accepted consensus with regard to second-line therapy in pancreatic cancer. Nevertheless, a growing number of studies suggest that patients can benefit from second-line therapy. In the CONKO-003 trial $[73,74]$, we randomly assigned patients to treatment with oxaliplatin, folinic acid and 5-FU (OFF) or best supportive care. Median survival in patients receiving second-line treatment with OFF was 4.82 months versus 2.30 months with best supportive care alone (HR 0.45, p $=0.008)$.

Meanwhile, subsequent phase II trials have further validated the value of fluoropyrimidine-based regimes in second-line treatment. Oxaliplatin plus capecitabine (XELOX) showed comparable efficacy in a phase II trial and offers the possibility of oral fluoropyrimidine treatment [75]. A recently presented randomized phase II trial [76] compared the most readily available fluoropyrimidine (5-FU, UFT or S-1) with continuation of gemcitabine. Fluoropyrimidine-treated patients benefited from a non-significantly prolonged progression-free survival of 113 days vs. 50 days $(\mathrm{p}=0.1050)$ and a significantly improved overall survival of 226 days vs. 161 days $(\mathrm{p}=$ 0.0384 ). The results of another randomized phase II trial suggests that the combination of a fluoropyrimidine with irinotecan could also be of value as second-line treatment [77]. Summing up the currently available evidence, patients with a good performance status who have progressed on gemcitabine-based therapy are recommended to receive second-line therapy consisting of oxaliplatin and an (oral) fluoropyrimidine. 
Table 2. Efficacy data in selected pivotal phase III Trials in patients with advanced disease.

\begin{tabular}{ccccc}
\hline Study & GEM [28] & GEM/ERL [33] & FOLFIRINOX [64] & NABPAC [71] \\
\hline ORR & $5.4 \%$ & $8.6 \%$ & $31.6 \%$ & $29 \%$ \\
OS & 5.65 months & 6.24 months & 11.1 months & 8.5 months \\
1-year OS & $18 \%$ & $23 \%$ & $48.4 \%$ & $35 \%$ \\
18-month OS & NR & NR & $18.6 \%$ & $16 \%$ \\
\hline
\end{tabular}

ORR, overall response rate; OS, overall survival; GEM, gemcitabine; ERL, erlotinib; NABPAC, nab-paclitaxel.

Table 3. Grade $\geq 3$ adverse events in selected pivotal phase III trials in patients with advanced disease.

\begin{tabular}{ccccc}
\hline Study & GEM [28] & GEM/ERL [33] & FOLFIRINOX [64] & NABPAC [71] \\
\hline Neutropenia & $25.9 \%$ & $24 \%$ & $45.7 \%$ & $38 \%$ \\
Febrile neutropenia & NR & NR & $5.4 \%$ & $3 \%$ \\
Thrombocytopenia & $9.7 \%$ & $10 \%$ & $9.1 \%$ & $13 \%$ \\
Fatigue & NR & $15 \%$ & $23.6 \%$ & $17 \%$ \\
Diarrhea & $1.6 \%$ & $6 \%$ & $12.7 \%$ & $6 \%$ \\
Peripheral neuropathy & $\mathrm{NR}$ & $\mathrm{NR}$ & $9.0 \%$ & $17 \%$ \\
\hline
\end{tabular}

ORR, overall response rate; OS, overall survival; GEM, gemcitabine; ERL, erlotinib; NABPAC, nab-paclitaxel.

\section{Prognostic and Predictive Factors in Pancreatic Cancer}

Currently, a lot of research is being conducted to identify and validate prognostic and predictive biomarkers in pancreatic cancer to help guide therapy decisions. Prognostic features predict prognosis independently from treatment and are based on the clinical features of the patient or the biological characteristics of pancreatic cancer such as tumor pathology whereas predictive factors predict tumor response to therapy [78]. An elevated CA 19-9 level has been identified as an independent negative prognostic biomarker in many trials. A recent comparison of the RTOG-9704 trial with the CONKO-001 trial showed that CA 19-9 values $\geq 90 \mathrm{U} / \mathrm{mL}$ after surgery were associated with a significantly shortened overall survival [79]. In the previously mentioned trial by Von Hoff et al. [71], CA19-9 decrease at 8 weeks predicted overall survival [80].

Another predictive factor in erlotinib-containing treatment regimens is the occurrence of a rash. In several phase III trials comparing erlotinib in combination with gemcitabine with gemcitabine alone in patients with unresectable advanced disease (AVITA, PA.3, AIO PK and PANTAR), occurrence of a treatment-related rash was indicative of response to erlotinib and resulted in significantly ( $p<0.001$ for AVITA, PA.3 and AIO PK; $p=$ 0.001 for PANTAR) improved survival, compared with those patients who did not develop a rash [81-84].

hENT1, (human equilibrative nucleoside transporter-1) has been identified as a potential predictor of overall survival in patients receiving gemcitabine [85]. Multivariate analysis of the adjuvant ESPAC-1 and -3 randomized trials confirmed increased intratumoural hENT1 expression as a predictive marker for response to gemcitabine (Wald $\chi^{2}=7.10, p=0.008$ ) but not fluorouracil (Wald $\chi^{2}$ $=0.34, \mathrm{p}=0.560$ ) [86]. Similar results were seen in the RTOG-9704 trial, in which higher hENT1 levels, upon multivariate analysis, were also correlated with improved overall survival in gemcitabine recipients (median of 24.2 vs. 14.8 months; $p=0.018$ ), but not in 5-FU recipients [87].

In a trial currently being conducted by Evans et al. (NCT01726582), feasibility of determining the most appropriate neoadjuvant treatment is being assessed in patients with borderline resectable disease. Six biomarkers have been selected based on their relevance to accepted pancreatic cancer chemotherapy regimens: high expression of SPARC (nab-paclitaxel), low expression of RRM1 (gemcitabine), low expression of ERCC1 (platinum analogs), high expression of TOPO1 (irinotecan), high expression of hENT1 (gemcitabine) and low expression of TYMS (fluorouracil). The primary endpoint is the resection rate and completion is scheduled for August 2014.

\section{Conclusion}

Currently, resection offers the best chance of cure in early-stage pancreatic cancer. Recent advances in adjuvant treatment have resulted in meaningful improvements in disease-free and overall survival, including in patients with an $\mathrm{R} 1$ resection or with previously unresectable disease, yet there is still no consensus on the optimal line of treatment. With several large phase III 
trials scheduled to be completed in the next years, including at least one investigating the emerging area of immunotherapy, hopefully the way forward will become better elucidated. Although progress has been made in the treatment of metastatic pancreatic cancer in the last few decades, it is clear that new strategies are needed if patients' lives are to be substantially extended and the realization is starting to emerge that targeting the primary tumor alone is not enough in the most resilient malignancies. The relatively recent recognition of the tumor microenvironment as a key player in tumor development and immune evasion and, moreover, that the stroma is a major factor in the notable drug resistance of pancreatic cancer, have marked the beginning of somewhat of a paradigm shift in the way pancreatic cancer and its treatments are viewed. New therapies, in which a multipronged approach is employed, targeting not only the primary tumor, but also the surrounding structures such as the tumor stroma, are starting to be explored with the hope of increasing response rates and subsequently improving survival outcomes. Nab-paclitaxel heralds the beginning of this new era, demonstrating significantly greater efficacy in advanced disease when administered with gemcitabine in the phase III setting than the current standard of care, gemcitabine monotherapy, and with less toxicity than FOLFIRINOX. It will be interesting to see if this proven efficacy in the palliative setting can be extrapolated to the adjuvant and neoadjuvant setting as well as in patients with locally advanced unresectable disease. Nab-paclitaxel should be considered as an eminent therapeutic option in combination with gemcitabine in the treatment of patients with pancreatic cancer.

\section{Acknowledgements}

The authors wish to express their gratitude to all of the participants who have taken part in clinical trials as well as to the study investigators, study nurses and data collectors and, finally, to the study groups and donors, all of whom have helped contribute to developing more effective therapies for pancreatic cancer. Researchers continue to search for new explanations, resulting in an increased understanding of pancreatic cancer biology and, ultimately, translation of this knowledge into novel approaches in the treatment of this challenging disease.

Medical writing assistance was provided by Dr. Marc Esser and Melody Watson at co.faktor (Berlin, Germany), funded by Celgene Corporation.

The author is fully responsible for all content and editorial decisions for this manuscript.

\section{REFERENCES}

[1] C. L. Wolfgang, J. M. Herman, D. A. Laheru, A. P. Klein, M. A. Erdek, et al., "Recent Progress in Pancreatic Can- cer," CA: A Cancer Journal for Clinicans, Vol. 63, No. 5, 2013, pp. 318-348. http://dx.doi.org/10.1002/caac.21190

[2] L. Lombardi, T. Troiano, N. Silvestris, L. Nanni, T. P. Latiano, et al., "Combined Modality Treatments in Pancreatic Cancer," Expert Opinion on Therapeutic Targets, Vol. 16, Suppl. 2, 2012, pp. S71-81.

http://dx.doi.org/10.1517/14728222.2012.662959

[3] R. A. Stathis and M. J. Moore, "Advanced Pancreatic Carcinoma: Current Treatment and Future Challenges," Nature Reviews. Clinical Oncology, Vol. 7, No. 3, 2010 , pp. 163-172.

http://dx.doi.org/10.1038/nrclinonc.2009.236

[4] M. Hidalgo, "Pancreatic Cancer," The New England Journal of Medicine, Vol. 362, No. 17, 2010, pp. 16051617. http://dx.doi.org/10.1056/NEJMra0901557

[5] A. Vincent, J. Herman, R. Schulick, R. H. Hruban and M. Goggins, "Pancreatic Cancer," Lancet, Vol. 378, No. 9791, 2001, pp. 607-620. http://dx.doi.org/10.1016/S0140-6736(10)62307-0

[6] M. M. Hassan, M. L. Bondy, R. A. Wolff, J. L. Abbruzzese, J. N. Vauthey, et al., "Risk Factors for Pancreatic Cancer: Case-Control Study," The American Journal of Gastroenterology, Vol. 102, No. 12, 2007, pp. 26962707.

http://dx.doi.org/10.1111/j.1572-0241.2007.01510.x

[7] C. Shi, H. R. Hruban and A. P. Klein, "Familial Pancreatic Cancer," Archives of Pathology \& Laboratory Medicine, Vol. 133, No. 3, 2009, pp. 365-374.

[8] A. Maitra and H. R. Hruban, "Pancreatic Cancer," Annual Review of Pathology: Mechanisms of Disease, Vol. 3, 2008, pp. 157-188.

http://dx.doi.org/10.1146/annurev.pathmechdis.3.121806. $\underline{154305}$

[9] S. Jones, X. Zhang, D. W. Parsons, J. C. Lin, R. J. Leary, et al., "Core Signaling Pathways in Human Pancreatic Cancers Revealed by Global Genomic Analyses," Science, Vol. 321, No. 5897, 2008, pp. 1801-1806. http://dx.doi.org/10.1126/science. 1164368

[10] G. Luo, J. Long, B. Zhang, C. Liu, J. Xu, et al., "Stroma and Pancreatic Ductal Adenocarcinoma: An Interaction Loop," Biochimica et Biophysica Acta, Vol. 1826, No. 1, 2012, pp. 170-178.

http://dx.doi.org/10.1016/j.bbcan.2012.04.002

[11] D. Mahadevan, D. D. Von Hoff, "Tumor-Stroma Interactions in Pancreatic Ductal Adenocarcinoma," Molecular Cancer Therapeutics, Vol. 6, No. 4, 2007, pp. 11861197. http://dx.doi.org/10.1158/1535-7163.MCT-06-0686

[12] M. V. Apte, S. Park, P. A. Phillips, N. Santucci, D. Goldstein, et al., "Desmoplastic Reaction in Pancreatic Cancer: Role of Pancreatic Stellate Cells," Pancreas, Vol. 29, No. 3, 2004, pp. 179-187. http://dx.doi.org/10.3389/fphys.2012.00344

[13] A. Masamune and T. Shimosegawa, "Signal Transduction in Pancreatic Stellate Cells," Journal of Gastroenterology, Vol. 44, No. 4, 2009, pp. 249-260. http://dx.doi.org/10.1007/s00535-009-0013-2

[14] M. Erkan, C. Reiser-Erkan, C. W. Michalski, S. Deucker, D. Sauliunaite, et al., "Cancer-Stellate Cell Interactions 
Perpetuate the Hypoxia-fibrosis Cycle in Pancreatic Ductal Adenocarcinoma," Neoplasia, Vol. 11, No. 5, 2009, pp. 497-508. http://dx.doi.org/10.1593/neo.81618

[15] N. P. Desai, V. Trieu, L. Y. Hwang, R. Wu, P. SoonShiong, et al., "Improved Effectiveness of Nanoparticle Albumin-bound (nab) Paclitaxel versus Polysorbate-Based Docetaxel in Multiple Xenografts as a Function of HER2 and SPARC Status," Anti-Cancer Drugs, Vol. 19, No. 9, 2008, pp. 899-909. http://dx.doi.org/10.1097/CAD.0b013e32830f9046

[16] C. J. Lee, J. Dosch and D. M. Simeone, "Pancreatic Cancer Stem Cells," Journal of Clinical Oncology, Vol. 26, No. 17, 2008, pp. 2806-2812.

http://dx.doi.org/10.1200/JCO.2008.16.6702

[17] P. C. Hermann, S. L. Huber, T. Herrler, A. Aicher, J. W. Ellwart, et al., "Distinct Populations of Cancer Stem Cells Determine Tumor Growth and Metastatic Activity in Human Pancreatic Cancer," Cell Stem Cell, Vol. 1, No. 3, 2007, pp. 313-323. http://dx.doi.org/10.1016/j.stem.2007.06.002

[18] G. Bond-Smith, N. Banga, T. M. Hammond and C. J. Imber, "Pancreatic Adenocarcinoma," BMJ, Vol. 344, 2012, Article ID: e2476. http://dx.doi.org/10.1136/bmj.e2476

[19] C. G. Moertel, D. S. Childs Jr., R. J. Reitemeier, M. Y. Colby Jr., M. A. Holbrook, et al., "Combined 5-fluorouracil and Supervoltage Radiation Therapy of Locally Unresectable Gastrointestinal Cancer," The Lancet, Vol. 2, No. 7626,1969 , pp. 865-867.

http://dx.doi.org/10.1016/S0140-6736(69)92326-5

[20] J. B. Haslam, P. J. Cavanaugh and S. L. Stroup, "Radiation Therapy in the Treatment of Irresectable Adenocarcinoma of the Pancreas," Cancer, Vol. 32, No. 6, 1973, pp. 1341-1345.

http://dx.doi.org/10.1002/1097-0142(197312)32:6<1341:: AID-CNCR2820320609>3.0.CO;2-A

[21] M. H. Kaiser and S. S. Ellenberg, "Pancreatic Cancer. Adjuvant combined Radiation and Chemotherapy Following Curative Resection," Archives of Surgery, Vol. 120, No. 8, 1985, pp. 899-903.

http://dx.doi.org/10.1001/archsurg.1985.01390320023003

[22] Gastrointestinal Tumor Study Group, "Further Evidence of Effective Adjuvant combined Radiation and Chemotherapy Following Curative Resection," Cancer, Vol. 59, No. 12,1987 , pp. 2006-2010.

http://dx.doi.org/10.1002/1097-0142(19870615)59:12<20 06::AID-CNCR2820591206>3.0.CO;2-B

[23] J. H. Klinkenbijl, J. Jeekel, T. Sahmoud, R. van Pel, M. L. Couvreur, et al., "Adjuvant Radiotherapy and 5-Fluorouracil after Curative Resection of Cancer of the Pancreas and Periampullary Region," Annals of Surgery, Vol. 230, No. 6, 1999, pp. 776-782. http://dx.doi.org/10.1097/00000658-199912000-00006

[24] H. G. Smeenk, C. H. J. van Eijck, W. C. Hop, J. Erdmann, K. C. K. Tran, et al., "Long-term Survival and Metastatic Pattern of Pancreatic and Periampullary Cancer after Adjuvant Chemoradiation or Observation: Long-term Results of EORTC trial 40891," Annals of Surgery, Vol. 246, No. 5, 2007, pp. 734-740.

\section{http://dx.doi.org/10.1097/SLA.0b013e318156eef3}

[25] J. P. Neoptolemos, D. D. Stocken, H. Friess, C. Bassi, J. A. Dunn, et al., "A Randomized Trial of Chemoradiotherapy and Chemotherapy after Resection of Pancreatic Cancer," The New England Journal of Medicine, Vol. 350, No. 12, 2004, pp. 1200-1210.

http://dx.doi.org/10.1056/NEJMoa032295

[26] H. Oettle, S. Post, P. Neuhaus, K. Gellert, J. Langrehr, et al., "Adjuvant Chemotherapy with Gemcitabine vs Observation in Patients Undergoing Curative-intent Resection of Pancreatic Cancer: A Randomized Controlled Trial.," JAMA: The Journal of the American Medical Association, Vol. 297, No. 3, 2007, pp. 267-277.

http://dx.doi.org/10.1001/jama.297.3.267

[27] J. P. Neoptolemos, D. D. Stocken, C. Bassi, P. Ghaneh, D. Cunningham, et al., "Adjuvant Chemotherapy with Fluorouracil plus Folinic Acid vs Gemcitabine Following Pancreatic Cancer Resection," JAMA: The Journal of the American Medical Association, Vol. 304, No. 10, 2010, pp. 1073-1081. http://dx.doi.org/10.1001/jama.2010.1275

[28] H. A. Burris 3rd, M. J. Moore, J. Andersen, M. R. Green, M. L. Rothenberg, et al., "Improvements in Survival and Clinical Benefit with Gemcitabine as First-line Therapy for Patients with Advanced Pancreas Cancer: A Randomized Trial," Journal of Clinical Oncology, Vol. 15, No. 6, 1997, pp. 2403-2413.

[29] W. F. Regine, K. A. Winter, R. A. Abrams, H. Safran, J. P. Hoffman, et al., "Fluorouracil vs Gemcitabine Chemotherapy before and after Fluorouracil-based Chemoradiation following Resection of Pancreatic Adenocarcinoma: A Randomized Controlled Trial," JAMA: The Journal of the American Medical Association, Vol. 299, No. 9, 2008, pp. 1019-26. http://dx.doi.org/10.1001/jama.299.9.1019

[30] Fukutomi, K. Uesaka, N. Boku, H. Kanemoto, M. Konishi, et al., "JASPAC 01: Randomized Phase III Trial of Adjuvant Chemotherapy with Gemcitabine versus S-1 for Patients with Resected Pancreatic Cancer," Journal of Clinical Oncology, Vol. 31, Suppl. 4003, 2013.

[31] D. D. Stocken, M. W. Büchler, C. Dervenis, C. Bassi, H. Jeekel, et al., "Meta-Analysis of Randomised Adjuvant Therapy Trials for Pancreatic Cancer," British Journal of Cancer, Vol. 92, No. 8, pp. 1372-1381.

http://dx.doi.org/10.1038/sj.bjc.6602513

[32] A. Vincent, J. Herman, R. Schulick, R. H. Hruban and M. Goggins, "Pancreatic Cancer," The Lancet, Vol. 378, No. 9791, 2001, pp. 607-620. http://dx.doi.org/10.1016/S0140-6736(10)62307-0

[33] M. J. Moore, D. Goldstein, J. Hamm, A. Figer, J. R. Hecht, et al., "Erlotinib plus Gemcitabine Compared with Gemcitabine alone in Patients with Advanced Pancreatic Cancer: A Phase III Trial of the National Cancer Institute of Canada Clinical Trials Group," Journal of Clinical Oncology, Vol. 25, No. 15, 2007, pp. 1960-1966. http://dx.doi.org/10.1200/JCO.2006.07.9525

[34] K. S. Gunturu, G. R. Rossi and M. W. Saif, "Immunotherapy Updates in Pancreatic Cancer: Are We There Yet?" Therapeutic Advances in Medical Oncology, Vol. 5, No. 1, 2013, pp. 81-89. 
http://dx.doi.org/10.1177/1758834012462463

[35] J. M. Hardacre, M. F. Mulcahy, et al., "Addition of Algenpantucel-L Immunotherapy to Standard of Care (SOC) Adjuvant Therapy for Pancreatic Cancer," Journal of Clinical Oncology, Vol. 30, Suppl. 4049, 2012.

[36] E. Lutz, C. J. Yeo, K. D. Lillemoe, B. Biedrzycki, B. Kobrin, et al., "A Lethally Irradiated Allogeneic Granulocyte-macrophage Colony Stimulating Factor-secreting Tumor Vaccine for Pancreatic Adenocarcinoma. A Phase II Trial of Safety, Efficacy, and Immune Activation," Annals of Surgery, Vol. 253, No. 2, 2011, pp. 328-335. http://dx.doi.org/10.1097/SLA.0b013e3181fd271c

[37] C. Belli, S. Cereda, S. Anand and M. Reni, "Neoadjuvant Therapy in Resectable Pancreatic Cancer: A Critical Review," Cancer Treatment Reviews, Vol. 29, No. 5, 2013, pp. 518-524. http://dx.doi.org/10.1016/j.ctrv.2012.09.008

[38] S. Heinrich, B. C. Pestalozzi, M. Schäfer, A. Weber, P. Bauerfeind, et al., "Prospective Phase II Trial of Neoadjuvant Chemotherapy with Gemcitabine and Cisplatin for Resectable Adenocarcinoma of the Pancreatic Head," Journal of Clinical Oncology, Vol. 26, No. 15, 2008, pp. 2526-2531. http://dx.doi.org/10.1200/JCO.2007.15.5556

[39] D. H. Palmer, D. D. Stocken, H. Hewitt, C. E. Markham, A. B. Hassan, et al., "A Randomized Phase 2 Trial of Neoadjuvant Chemotherapy in Resectable Pancreatic Cancer: Gemcitabine Alone versus Gemcitabine combined with Cisplatin," Annals of Surgical Oncology, Vol. 14, No. 7, 2007, pp. 2088-2089. http://dx.doi.org/10.1245/s10434-007-9384-x

[40] M. S. Talamonti, W. Small Jr., M. F. Mulcahy, J. D. Wayne, V. Attaluri, et al., "A Multi-Institutional Phase II Trial of Preoperative Full-dose Gemcitabine and Concurrent Radiation for Patients with Potentially Resectable Pancreatic Carcinoma," Annals of Surgical Oncology, Vol. 13, No. 7, 2006, pp. 150-158. http://dx.doi.org/10.1245/ASO.2006.03.039

[41] G. R. Varadhachary, R. A. Wolff, C. H. Crane, C. C. Sun, J. E. Lee, et al., "Preoperative Gemcitabine and Cisplatin Followed by Gemcitabine-Based Chemoradiation for Resectable Adenocarcinoma of the Pancreatic Head," Journal of Clinical Oncology, Vol. 26, 2008, pp. 3487-3495. http://dx.doi.org/10.1200/JCO.2007.15.8642

[42] S. Gillen, T. Schuster, C. Meyer Zum Büschenfelde, H. Friess and J. Kleeff, "Preoperative/Neoadjuvant Therapy in Pancreatic Cancer: A Systematic Review and MetaAnalysis of Response and Resection Percentages," PLoS Medicine, Vol. 7, No. 4, 2010. http://dx.doi.org/10.1371/journal.pmed.1000267

[43] S. MacKenzie, H. Zeh, L. E. McCahill, T. D, Sielaff, N. Bahary, et al., "A Pilot Phase II Multicenter Study of Nab-paclitaxel (Nab-P) and Gemcitabine (G) as Preoperative Therapy for Potentially Resectable Pancreatic Cancer (PC)," Journal of Clinical Oncology, Vol. 31 Suppl. 4038, 2013.

[44] D. Habermehl, K. Kessel, T. Welzel, H. Hof, A. Abdollahi, et al., "Neoadjuvant Chemoradiation with Gemcitabine for Locally Advanced Pancreatic Cancer," Journal of Radiation Oncology, Vol. 7, No. 28, 2012, p. 28. http://dx.doi.org/10.1186/1748-717X-7-28
[45] M. Nakamura, T. Kayashima, K. Fujiwara, Y. Nagayoshi and H. Kono, "Combination Therapy of Portal Vein Resection and Adjuvant Gemcitabine Improved Prognosis of Advanced Pancreatic Cancer," Hepatogastroenterology, Vol. 60, No. 122, 2013, pp. 354-357. http://dx.doi.org/10.5754/hge12614

[46] B. Chauffert, F. Mornex, F. Bonnetain, P. Rougier and C. Mariette, "Phase III Trial Comparing Intensive Induction Chemoradiotherapy (60 Gy, Infusional 5-FU and Intermittent Cisplatin) Followed by Maintenance Gemcitabine with Gemcitabine Alone for Locally Advanced Unresectable Pancreatic Cancer. Definitive Results of the 2000-01 FFCD/SFRO Study," Annals of Oncology, Vol. 19, No. 9, 2008, pp. 1592-1599.

http://dx.doi.org/10.1093/annonc/mdn281

[47] P. J. Loehrer Sr, Y. Feng, H. Cardenes, L. Wagner, J. M. Brell, et al., "Gemcitabine Alone versus Gemcitabine plus Radiotherapy in Patients With Locally Advanced Pancreatic Cancer: An Eastern Cooperative Oncology Group Trial," Journal of Clinical Oncology, Vol. 29, No. 31, 2011, pp. 4105-4112.

http://dx.doi.org/10.1200/JCO.2011.34.8904

[48] S. Mukherjee, C. Hurt, G. Griffiths, J. A. Bridgewater, T. Crosby, et al., "SCALOP: Results of a Randomized Phase II Study of Induction Chemotherapy Followed by Gemcitabine $(\mathrm{G})$ or Capecitabine (Cap) based Chemoradiation (CRT) in Locally Advanced Pancreatic Cancer (LAN PC)," Journal of Clinical Oncology, Vol. 30, Suppl. 34, 2012.

[49] P. Hammel, F. Huguet, J-L. Van Laethem, D. Goldstein, B. Glimelius, et al., "Comparison of Chemoradiotherapy (CRT) and Chemotherapy (CT) in Patients with a Locally Advanced Pancreatic Cancer (LAPC) Controlled after 4 Months of Gemcitabine with or without Erlotinib: Final Results of the International Phase III LAP 07 study," Journal of Clinical Oncology, Vol. 31, Suppl. LBA4003, 2013.

[50] J. M. Herman, A. T. Wild, H. Wang, P. T. Tran, K. J. Chang, et al., "Randomized Phase III Multi-Institutional Study of TNFerade Biologic with Fluorouracil and Radiotherapy for Locally Advanced Pancreatic Cancer," Journal of Clinical Oncology, Vol. 31, 2013, pp. 886-894. http://dx.doi.org/10.1200/JCO.2012.44.7516

[51] J. D. Berlin, P. Catalano, J. P. Thomas, J. W Kugler, D. G. Haller, et al., "Phase III Study of Gemcitabine in Combination with Fluorouracil versus Gemcitabine alone in Patients with Advanced Pancreatic Carcinoma: Eastern Cooperative Oncology Group Trial E2297," Journal of Clinical Oncology, Vol. 20, No. 15, 2002, pp. 3270-3275. http://dx.doi.org/10.1200/JCO.2002.11.149

[52] R. Herrmann, G. Bodoky, T. Ruhstaller, B. Glimelius, E. Bajetta E, et al., "Gemcitabine plus Capecitabine Compared with Gemcitabine alone in Advanced Pancreatic Cancer: A Randomized, Multicenter, Phase III Trial of the Swiss Group for Clinical Cancer Research and the Central European Cooperative Oncology Group," Journal of Clinical Oncology, Vol. 25, No. 16, 2007, pp. 22122217. http://dx.doi.org/10.1200/JCO.2006.09.0886

[53] C. Louvet, R. Labianca, P. Hammel, G. Lledo, M. G. Zampino, et al., "Gemcitabine in Combination with Ox- 
aliplatin Compared with Gemcitabine alone in Locally Advanced or Metastatic Pancreatic Cancer: Results of a GERCOR and GISCAD phase III Trial," Journal of Clinical Oncology, Vol. 23, No. 15, 2005, pp. 3509-3516. http://dx.doi.org/10.1200/JCO.2005.06.023

[54] V. Heinemann, D Quietzsch, F. Gieseler, M. Gonnermann, H. Schönekäs, et al., "Randomized Phase III trial of Gemcitabine plus Cisplatin compared with Gemcitabine alone in Advanced Pancreatic Cancer," Journal of Clinical Oncology, Vol. 24, No. 24, 2006, pp. 3946-3952. http://dx.doi.org/10.1200/JCO.2005.06.023

[55] C. M. Rocha Lima, M. R Gree, R Rotche, W. H. Miller, G. M. Jeffrey, et al., "Irinotecan plus Gemcitabine Results in no Survival Advantage Compared with Gemcitabine Monotherapy in Patients with Locally Advanced or Metastatic Pancreatic Cancer despite Increased Tumor Response Rate," Journal of Clinical Oncology, Vol. 22, No. 18, 2004, pp. 3776-3783. http://dx.doi.org/10.1200/JCO.2004.12.082

[56] H. Oettle, D. Richards, R. K. Ramanathan, J. L. van Laethem, M. Peeters, et al., "A Phase III Trial of Pemetrexed plus Gemcitabine versus Gemcitabine in $\mathrm{Pa}$ tients with Unresectable or Metastatic Pancreatic Cancer," Annals of Oncology, Vol. 16, No. 10, 2005 pp. 16391645. http://dx.doi.org/10.1093/annonc/mdi309

[57] A. Sultana, C. T. Smith, D. Cunningham, N. Starling, J. P. Neoptolemos, et al., "Meta-analyses of Chemotherapy for Locally Advanced and Metastatic Pancreatic Cancer". Journal of Clinical Oncology, Vol. 25, No. 18, 2007, pp. 2607-1265. http://dx.doi.org/10.1200/JCO.2006.09.2551

[58] V. Heinemann, S. Boeck, A. Hinke, R. Labianca, C. Louvet, et al., "Meta-Analysis of Randomized Trials: Evaluation of Benefit from Gemcitabine-based Combination Chemotherapy Applied in Advanced Pancreatic Cancer," BMC Cancer, Vol. 8, 2008, p. 82. http://dx.doi.org/10.1186/1471-2407-8-82

[59] E. van Cutsem, W. L. Vervenne, J. Bennouna, Y. Humblet Y, S. Gill, et al., "Phase III Trial of Bevacizumab in Combination with Gemcitabine and Erlotinib in Patients with Metastatic Pancreatic Cancer," Journal of Clinical Oncology, Vol. 27, No. 13, 2009 pp. 2231-2237. http://dx.doi.org/10.1200/JCO.2008.20.0238

[60] H. L. Kindler, D. Niedzwiecki, D. Hollis, S. Sutherland, D. Schrag, et al., "Gemcitabine plus Bevacizumab Compared with Gemcitabine plus Placebo in Patients with Advanced Pancreatic Cancer: Phase III Trial of the Cancer and Leukemia Group B (CALGB 80303)," Journal of Clinical Oncology, Vol. 28, No. 22, pp. 3617-3622. http://dx.doi.org/10.1200/JCO.2010.28.1386

[61] P. A. Philip, J. Benedetti, C. L. Corless, R. Wong, E. M. O'Reill, et al., "Phase III Study Comparing Gemcitabine plus Cetuximab versus Gemcitabine in Patients with Advanced Pancreatic Adenocarcinoma: Southwest Oncology Group-directed Intergroup Trial S0205," Journal of Clinical Oncology, Vol. 28, No. 22, 2010, pp. 3605-3610. http://dx.doi.org/10.1200/JCO.2009.25.7550

[62] J. P. Spano, C. Chodkiewicz, J. Maurel, R. Wong, H. Wasan, et al., "Efficacy of Gemcitabine Plus Axitinib Compared with Gemcitabine Alone in Patients with Ad- vanced Pancreatic Cancer: An Open-label Randomised phase II Study," Lancet, Vol. 371, No. 9630, pp. 21012108. http://dx.doi.org/10.1016/S0140-6736(08)60661-3

[63] A. Gonçalves, M. Gilabert, E. François, L. Dahan, H. Perrier, et al., "BAYPAN Study: A Double-blind Phase III Randomized Trial Comparing Gemcitabine plus Sorafenib and Gemcitabine plus Placebo in Patients with Advanced Pancreatic Cancer," Annals of Oncology, Vol. 23, No 11, pp. 2799-2805.

http://dx.doi.org/10.1093/annonc/mds135

[64] T. Conroy, F. Desseigne, M. Ychou, O. Bouché, R. Guimbaud, et al., "FOLFIRINOX versus Gemcitabine for Metastatic Pancreatic Cancer," The New England Journal of Medicine, Vol. 364, No. 19, pp. 1817-1825. http://dx.doi.org/10.1056/NEJMoa1011923

[65] P. J. Hosein, J. Macintyre, C. Kawamura, J. C. Maldonado, V. Ernani, et al., "A Retrospective Study of Neoadjuvant FOLFIRINOX in Unresectable or BorderlineResectable Locally Advanced Pancreatic Adenocarcinoma," BMC Cancer, Vol. 12, No. 199, 2012. http://dx.doi.org/10.1186/1471-2407-12-199

[66] T. Conroy, C. Gavoille, E. Samalin, M. Ychou, M. Ducreux, et al., "The Role of the FOLFIRINOX Regimen for Advanced Pancreatic Cancer," Current Oncology Reports, Vol. 15, No. 2, 2013, pp. 182-189. http://dx.doi.org/10.1007/s11912-012-0290-4

[67] A. Neesse, P. Michl, K. K. Frese, C. Feig, N. Cook, et al., "Stromal Biology and Therapy in Pancreatic Cancer," Gut, Vol. 60, No. 6, 2011, pp. 861-868. http://dx.doi.org/10.1136/gut.2010.226092

[68] J. R. Infante, H. Matsubayashi, N. Sato, J. Tonascia, A. P. Klein, et al., "Peritumoral Fibroblast SPARC Expression and Patient Outcome with Resectable Pancreatic Adenocarcinoma," Journal of Clinical Oncology, Vol. 25, No. 3, 2007, pp. 319-325. http://dx.doi.org/10.1200/JCO.2006.07.8824

[69] V. Trieu, J. Hwang and N. Desai, "Nanoparticle Albuminbound (nab) Technology may Enhance Antitumour Activity via Targeting of SPARC Protein," Proceedings New Targets and Delivery System for Cancer Diagnosis and Treatment conference, Sidney Kramer Cancer Center, San Diego, 5-7 March 2007.

[70] D. D. Von Hoff, R. K. Ramanathan, M. J. Borad, D. A. Laheru, L. S. Smith, et al., "Gemcitabine Plus Nab-Paclitaxel is an Active Regimen in Patients with Advanced Pancreatic Cancer: A Phase I/II Trial," Journal of Clinical Oncology, Vol. 29, No. 34, 2011, pp. 4548-4554. http://dx.doi.org/10.1200/JCO.2011.36.5742

[71] D. D. von Hoff, T. J. Ervin, F. P. Arena, E. G. Chiorean, R. J. Infante, et al., "Randomized Phase III Study of Weekly Nab-Paclitaxel plus Gemcitabine versus Gemcitabine alone in Patients with Metastatic Adenocarcinoma of the Pancreas (MPACT)," Journal of Clinical Oncology, Vol. 30, Suppl. 34, 2012

[72] M. J. Moore, D. D. Von Hoff, T. J. Ervin, F. P. Arena, E. G. Chiorean, et al., "Prognostic Factors (PFs) of Survival in a Randomized Phase III Trial (MPACT) of Weekly Nab-paclitaxel (nab-P) plus Gemcitabine (G) versus G alone in Patients (pts) with Metastatic Pancreatic Cancer 
(MPC)," Journal of Clinical Oncology, Vol. 31, Suppl. 4059, 2013.

[73] U. Pelzer, K. Kubica, J. Stieler, I. Schwaner, G. Heil, et al., "A Randomized Trial in Patients with Gemcitabine Refractory Pancreatic Cancer. Final Results of the CONKO 003 Study," Journal of Clinical Oncology, Vol. 26, No. 15S, Suppl. 4508, 2008.

[74] U. Pelzer, I. Schwaner, J. Stieler, M. Adler, J. Seraphin J., et al., "Best Supportive Care (BSC) versus Oxaliplatin, Folinic Acid and 5-Fluorouracil (OFF) Plus BSC in Patients for Second-line Advanced Pancreatic Cancer: A Phase III-study from the German CONKO-Study Group," European Journal of Cancer, Vol. 47, No. 11, 2011, pp. 1676-1681. http://dx.doi.org/10.1016/j.ejca.2011.04.011

[75] H. Q. Xiong, G. R. Varadhachary, J. C. Blais, K. R. Hess, J. L. Abbruzzese, et al., "Phase 2 Trial of Oxaliplatin plus Capecitabine (XELOX) as Second-line therapy for Patients with Advanced Pancreatic Cancer," Cancer, Vol. 113, No. 8, 2008, pp. 2046-2052. http://dx.doi.org/10.1002/cncr.23810

[76] N. Mizuno, K. Yamao, Y. Komatsu, M. Munakata, A. Ishiguro, et al., "Randomized Phase II Study of Best Available Fluoropyrimidine Compared with Continuation of Gemcitabine (Gem) Monotherapy in Patients with Gem-Refractory Pancreatic Cancer," Journal of Clinical Oncology, Vol. 30, Suppl. 34, Abstr. 263, 2012.

[77] T. Ioka, K. Katayama, N. Ishida, R. Takada, T. Yamai, et al., "Randomized Phase II Trial of S-1 versus S-1 Plus Irinotecan (IRIS) in Patients with Gemcitabine-Refractory Pancreatic Cancer," Journal of Clinical Oncology, Vol. 30, Suppl. 34, Abstr. 287, 2012.

[78] C. N. Oldenhuis, S. F. Oosting, J. A. Gietema and E. G. de Vries, "Prognostic versus Predictive Value of Biomarkers in Oncology," European Journal of Cancer, Vol. 44, No. 7, 2008, pp. 946-953. http://dx.doi.org/10.1016/j.ejca.2008.03.006

[79] A. C. Berger, K. Winter, J. P. Hoffman, W. F. Regine, R. A. Abrams, et al., "Five Year Results of US Intergroup/ RTOG 9704 with Postoperative CA 19-9 $\leqslant 90$ U/mL and Comparison to the CONKO-001 Trial," International Journal of Radiation Oncology Biology Physics, Vol. 84, No. 3, 2012, pp. 291-297. http://dx.doi.org/10.1016/j.ijrobp.2012.04.035

[80] E. G. Chiorean, D. D. Von Hoff, T. J. Ervin, F. P. Arena, J. R. Infante, et al., "CA19-9 Decrease at 8 Weeks as a Predictor of Overall Survival (OS) in a Randomized Phase III Trial (MPACT) of Weekly Nab-paclitaxel (nabP) plus Gemcitabine $(\mathrm{G})$ versus $G$ alone in Patients with
Metastatic Pancreatic Cancer (MPC)," Journal of Clinical Oncology, Vol. 31, Suppl. 4058, 2013.

[81] C. Verslype, W. Verwvenne, J. Bennouna, Y. Humblet, J. Cosaert J, et al., "Rash as a Marker for the Efficacy of Gemcitabine plus Erlotinib-based Therapy in Pancreatic Cancer: Results from the AViTA Study," Journal of Clinical Oncology, Vol. 27, Suppl. 4532, 2009.

[82] J. Manzano, F. Rivera, M. Gala, M. Valladares, C. Pericay, et al., "A Phase II, Open Label Study to Evaluate the Relationship between Skin Rash and Survival in Patients with Unresectable and/or Metastatic Pancreatic Cancer Treated with Erlotinib combined with Gemcitabine," Journal of Clinical Oncology, Vol. 28, Abstr. 4094.

[83] E. Aranda, J. L. Manzano, F. Rivera, M. Galán, M. Valladares-Ayerbes, et al., "Phase II Open-label Study of Erlotinib in Combination with Gemcitabine in Unresectable and/or Metastatic Adenocarcinoma of the Pancreas: Relationship between Skin Rash and Survival (Pantar Study)," Annals of Oncology, Vol. 23, No. 7, 2012, pp. 1919-1925. http://dx.doi.org/10.1093/annonc/mdr560

[84] V. Heinemann, U. Vehling-Kaiser, D. Waldschmidt, E. Kettner, A. Märten, et al., "Gemcitabine Plus Erlotinib followed by Capecitabine versus Capecitabine plus Erlotinib followed by Gemcitabine in Advanced Pancreatic Cancer: Final Results of a Randomised Phase 3 Trial of the 'Arbeitsgemeinschaft Internistische Onkologie' (AIOPK0104)," Gut, Vol. 62, No. 5, 2013, pp. 751-762. http://dx.doi.org/10.1136/gutjnl-2012-302759

[85] J. García-Manteiga, M. Molina-Arcas, F. J. Casado, A. Mazo and M. Pastor-Anglada, "Nucleoside Transporter Profiles in Human Pancreatic Cancer Cells: Role of hCNT1 in 2',2'-Difluorodeoxycytidine-Induced Cytotoxicity," Clinical Cancer Research, Vol. 9, No. 13, 2003, pp. 5000-5008.

[86] J. P. Neoptolemos, W. Greenhalf, P. Ghaneh, D. H. Palmer, T. F. Cox, et al., "HENT1 Tumor Levels to Predict Survival of Pancreatic Ductal Adenocarcinoma Patients who Received Adjuvant Gemcitabine and Adjuvant 5FU on the ESPAC Trials," Journal of Clinical Oncology, Vol. 31, Suppl. 4006, 2013.

[87] J. J. Farrell, H. Elsaleh, M. Garcia, R. Lai, A. Ammar, et al., "Human Equilibrative Nucleoside Transporter 1 Levels Predict Response to Gemcitabine in Patients with Pancreatic Cancer," Gastroenterology, Vol. 136, No. 1, 2009, pp. 187-195.

http://dx.doi.org/10.1053/j.gastro.2008.09.067 